\title{
Sequence Reactivation in the Hippocampus Is Impaired in Aged Rats
}

\author{
Jason L. Gerrard, ${ }^{2}$ Sara N. Burke, ${ }^{1,2}$ Bruce L. McNaughton, ${ }^{1,2,3,5}$ and Carol A. Barnes ${ }^{1,2,3,4}$ \\ ${ }^{1}$ Evelyn F. McKnight Brain Institute, ${ }^{2}$ Arizona Research Laboratories Division of Neural Systems, Memory and Aging, and Departments of ${ }^{3}$ Psychology, \\ ${ }^{4}$ Neurology, and ${ }^{5}$ Physiology, University of Arizona, Tucson, Arizona 85724
}

The hippocampus is thought to coordinate memory consolidation by reactivating traces from behavioral experience when the brain is not actively processing new input. In fact, during slow-wave sleep, the patterns of CA1 pyramidal cell ensemble activity correlations are reactivated in both young and aged rats. In addition to correlated activity patterns, repetitive track running also creates a recurring sequence of pyramidal cell activity. The present study compared CA1 sequence activity pattern replay in young and old animals during rest periods after behavior. Whereas the young rats exhibited significant sequence reactivation, it was markedly impaired in the aged animals. When the spatial memory scores of all animals were compared with the degree of sequence reactivation, there was a significant correlation. The novel finding that weak replay of temporal patterns has behavioral consequences, strengthens the idea that reactivation processes are integral to memory consolidation.

Key words: aging; attractor dynamics; CA1; memory consolidation; place field; neural ensemble

\section{Introduction}

Clinical and experimental studies indicate that the hippocampus plays a critical role in the initial acquisition and consolidation of certain types of memory (Scoville and Milner, 1957; Winocur, 1990; Zola-Morgan and Squire, 1990; Bolhuis et al., 1994; Takehara et al., 2002, 2003). Although the specific nature of the hippocampal-neocortical interaction involved in memory consolidation remains unknown, it has been suggested that the hippocampus acts as an autoassociator, in which memories are spontaneously reactivated when the brain is not actively processing external inputs (Marr, 1971). In this view, during "off-line" periods such as sleep, the hippocampus would spontaneously replay activity patterns stored during previous experiences. This replay would then coordinate the recall of the related activity within multiple neocortical networks via the widely diverging hippocampal efferents. Such a process would allow the neocortex to form new categories or event classes, or to strengthen old structures as the new memory is incorporated into longer-term memory stores (for review, see Sutherland and McNaughton, 2000; McNaughton et al., 2003).

Since the advent of multineuron ensemble recordings (Wilson and McNaughton, 1994), empirical support for off-line ac-

\footnotetext{
Received March 24, 2008; revised May 8, 2008; accepted May 21, 2008.

This work was supported by National Institute on Aging Grant AG012609, the Arizona Chapter of the Achievement Rewards for College Scientists Foundation, and The McKnight Brain Research Foundation. We thank A. David Redish, Ephron Rosenzweig, Hemant Kudrimoti, Arne Ekstrom, Stephen Cowen, Mark Bower, Kate Poneta, Peter Lipa, Joe Bohanick, and Andrew Maurer for their help with various aspects of the data collection, analysis, and manuscript preparation. Additionally, we thank Luann Synder and Michelle Carroll for their administrative assistance.

Correspondence should be addressed to Dr. Carol A. Barnes, Life Sciences North Building, Room 384, The Evelyn

F. McKnight Brain Institute, University of Arizona, Tucson, AZ 85724. E-mail: carol@nsma.arizona.edu. DOI:10.1523/JNEUROSCI.1265-08.2008

Copyright $\odot 2008$ Society for Neuroscience $\quad 0270-6474 / 08 / 287883-08 \$ 15.00 / 0$
}

tivity pattern reactivation within the hippocampus (Wilson and McNaughton, 1994; Shen et al., 1998; Kudrimoti et al., 1999; Lee and Wilson, 2002) and neocortex (Qin et al., 1997; Hoffman and McNaughton, 2002; Euston et al., 2007; Ji and Wilson, 2007) has accumulated. In the hippocampus, reactivation occurs during periods of high synchronization (Kudrimoti et al., 1999; Nadasdy et al., 1999; Gerrard et al., 2001) that manifest in the electroencephalogram as "sharp waves" (Buzsáki, 1986). It has been suggested that sharp waves may reflect the spontaneous convergence of the CA3 network onto attractor (i.e., stored memory) states (Shen and McNaughton, 1996; Buzsáki, 1998), which would enable the hippocampus to "orchestrate" a memory consolidation process.

Aged animals show learning and memory deficits on hippocampal-dependent tasks (Barnes, 1979; Markowska et al., 1989; Gallagher and Rapp, 1997), as well as stimuli-induced alterations in long-term potentiation and long-term depression (for review, see Burke and Barnes, 2006), and impairments in behaviorally induced place field expansion plasticity (Shen et al., 1997). These findings suggest that aged animals may not be able to reactivate newly formed memory traces as robustly or accurately as young animals. Reactivation can be quantified both by measuring the correlations of activity patterns, and comparing the correlations established during behavior to the correlations during the subsequent sleep episode, or by comparing the temporal order of those activity patterns between behavior and sleep. Interestingly, the reactivation of simple correlated activity patterns, in the CA1 subregion of the hippocampus, is comparable between young and aged rats when the relative temporal order of cell pair firing was not taken into account (Gerrard et al., 2001). Because aged rats do not show a form of behaviorally induced plasticity (Shen et al., 1997) that is thought to reflect encoding of sequential information (Mehta et al., 2000), the present experi- 
ment tested the hypothesis that aged rats would not exhibit accurate reactivation of activity sequences.

\section{Materials and Methods \\ Subjects and behavioral training \\ A total of 22 male rats, 11 young (11-12 months) and 11 old (25-31 months) male Fischer 344 rats (from the colony of the National Institute on Aging at Harlan) were used in the experiments. The rats were housed individually in Plexiglas guinea pig tubs and maintained on a reversed $12 \mathrm{~h}$ light/dark cycle. Before beginning the electrophysiological portion of the experiments, the spatial and visual discrimination abilities of each rat were tested on the Morris swim task (for details, see Shen and Barnes, 1996). Briefly, the rats were tested on six spatial trials (hidden platform) per day for 4 consecutive days, followed by six visual discrimination trials in which the platform was visible above the water line. The visual dis- crimination portion of the procedure was used to assess the visual and motor functions of each rat, to ensure that visual or physical limitations did not impact the performance of the animals. Latency measures, rather than path length, were used in the present analysis because part of the tracking data were lost as a result of a disk malfunction. Latency data were available for all rats and were thus used here. Because the latency did not differ on cued trials, the swimming velocity between age groups was comparable. \\ Throughout the training and recording period, the rats were main- tained at $80 \%$ of their ad libitum body weights, had ad libitum access to water, and were handled and weighed daily. Before surgical implantation of the recording array, the animals were trained to run back and forth along a linear track to familiarize them with track-running behavior. During the initial 2-3 d of training on the linear track, rats were allowed to search for chocolate sprinkles scattered randomly along the entire length of the track. For the following weeks, the chocolate food reward was only available at the two ends of the linear track. Finally, the rats were familiarized with the individual experimental procedures as detailed be- low. On completion of behavioral training, electrodes were surgically implanted.}

\section{Surgery, electronics, and recording}

To acquire extracellular spike signals from multiple single cells, a multielectrode microdrive assembly or "hyperdrive" (Gothard et al., 1996) consisting of 14 independently movable "tetrode" record probes (McNaughton et al., 1983a; O'Keefe and Recce, 1993) was surgically implanted unilaterally above the right hippocampus of each rat. Each hyperdrive consisted of 14 drive screws coupled by a nut to a guide cannula. Twelve of these guide cannulas contained tetrodes that were constructed by twisting together four strands of insulated $13 \mu \mathrm{m}$ nichrome wire (H. P. Reid). Two additional tetrodes with their individual wires shorted together served as an indifferent reference and an EEG recording probe. A full turn of the screw advanced the tetrode $318 \mu \mathrm{m}$.

The National Institutes of Health and University of Arizona Institutional Animal Care and Use Committee guidelines were followed for all surgical procedures. Rats were anesthetized with Nembutal (sodium pentobarbital) at $33 \mathrm{mg} / \mathrm{kg}$, which was supplemented as necessary with inhaled Metofane (methoxyflurane). The hyperdrive array was stereotaxically positioned $3.8 \mathrm{~mm}$ posterior and $2.5 \mathrm{~mm}$ lateral to bregma, in a craniotomy drilled above the CA1 region of the right hippocampus (Paxinos and Watson, 1998). The implant was cemented in place with dental acrylic anchored by small screws. At surgery, all 14 of the probes were lowered $\sim 1200 \mu \mathrm{m}$ into the cortex. After surgery, the probes were lowered incrementally to their desired locations in the CA1 stratum pyramidale as the animal continued training for the experimental paradigm. Additionally, the neutral reference electrode was positioned in or near the corpus callosum. The four channels of each tetrode were attached to a 50-channel unity-gain headstage (Neuralynx). A multiwire cable connected the headstage to digitally programmable amplifiers (Neuralynx). The spike signals were amplified by a factor of 1000-5000, bandpass filtered between $600 \mathrm{~Hz}$ and $6 \mathrm{kHz}$, and transmitted to the Cheetah Data Acquisition system (Neuralynx). Signals were digitized at $32 \mathrm{kHz}$ and events that reached a predetermined threshold were recorded for a duration of $1 \mathrm{~ms}$.

\section{Behavioral procedures}

The animals in the present investigation participated in one of three track-running procedures. Although the tracks are different shapes, for the purposes of this investigation, each track and experimental paradigm involved the animal traversing the track repeatedly, either in one direction continuously or in a back-and-forth sequence from a particular starting point. In each experimental paradigm, the animal received either food or medial forebrain bundle (MFB) stimulation reward at specific points along the path of the track. Each of the three experimental procedures followed the same basic structure. To initiate each experimental recording session, the animal was placed into a nest pot and allowed to sleep/rest quietly for 30-60 min (REST1). After this, the animal was removed from the nest pot and placed onto one of three different tracks and ran several laps on the particular track used (RUN). These tracks and their associated behavioral procedures are described below. After track running was completed, the rats were placed back into the nest pot, in the training room, in which they were allowed to sleep or rest quietly for an additional 30-60 $\mathrm{min}$ (REST2). The data presented here all followed the procedure of a baseline rest period (REST1), a track-running period (RUN), and finally a rest period after the track-running epoch (REST2). All experiments were performed in "batches" that consisted of one young and one old animal. Specifically, during all electrophysiological recordings, the young and the aged rats were paired such that data were collected from one young rat and one aged rat during the same time period (i.e., time of year, over the same number of months, with the same experimenters). This type of design ensures that during any given experimental interval any uncontrolled variables would be experienced equivalently in subjects from both the young and old age groups.

Procedure A. The animals in this experiment repeatedly traversed a rectangular track in the counterclockwise direction, receiving food reward at two of the corners (Fig. 1A). Data from this subset of animals have been published previously in a report of preserved pattern reactivation in aged rats (Gerrard et al., 2001).

Procedure B. After the initial REST1 epoch, the animals in this paradigm were placed at the base arm of a T-shaped track. The animals traversed back and forth along the T-shaped track, receiving food reward at the ends of the track (Fig. $1 B$ ). During the track-running period (RUN), the animal received food reward at the two ends of the T-shaped track with different probabilities ( 80 vs $20 \%$ ) and the base arm was always baited $50 \%$ of the time. Thus, it was possible for the animal to learn which end arm of the T-shaped track was more likely to possess food reward. During $80 \%$ of the laps, however, a barrier was placed at the intersection of the " $\mathrm{T}$," allowing the animal to turn in only one of the directions. This provided equal sampling of the entire track to establish place cell-firing characteristics. During the other $20 \%$ of the laps, no barrier was in place and the animal was allowed to choose between the two arms. The results of these probe trials were not used in the present experiment.

Procedure C. Animals were implanted with an additional stimulating electrode placed into the MFB, and small amounts of current were delivered to serve as a reward (Olds and Milner, 1954). During the trackrunning portion of this paradigm, the animals were placed within an enclosed box that was on a circular track that could be rotated to increase or decrease the distance from the start box to the end of the track (Fig. $1 C)$. There was a stable barrier over one portion of the track, which demarcated the end of the track. In addition, there was an unmarked portion of the track in which the animal could receive MFB stimulation if he paused for a brief time period within that location. The animals ran back and forth from the start box to the barrier and then back to the start box, with the possibility of receiving MFB stimulation in each direction. In addition, the animals received food reward within the start box after the completion of each trial. The data regarding the effects of changing the track length and MFB stimulation were presented previously (for details, see Redish et al., 2000; Rosenzweig et al., 2003). For the purposes of the present experiment, the track running (RUN) and the rest epochs (REST1 and REST2) were taken en bloc, without any parsing of outbound versus inbound journeys or the MFB stimulation location. 
A

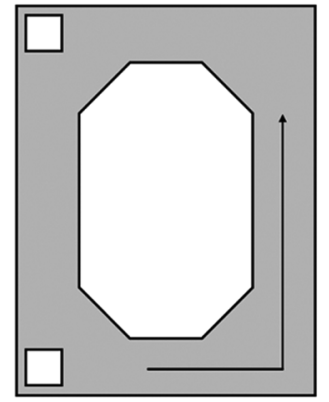

B

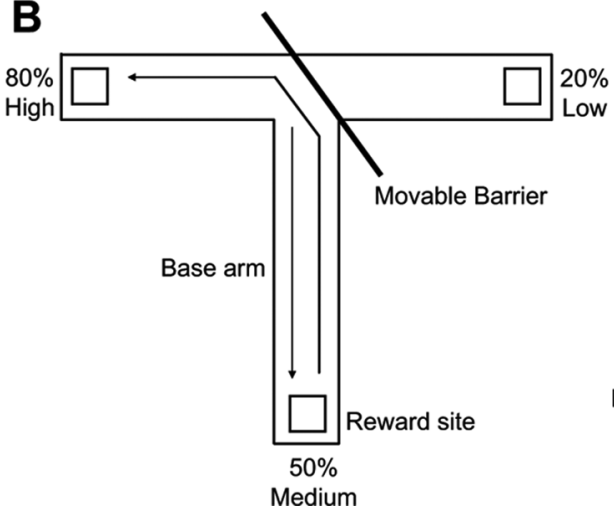

C

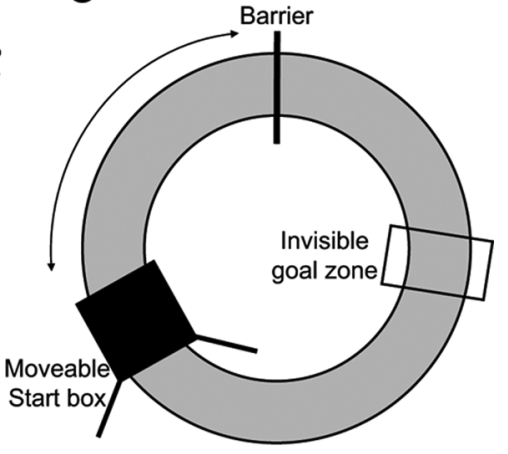

Figure 1. Apparati used in the three behavioral procedures from which data in the present experiment were obtained. $A$, Rectangular track. Rats traversed a rectangular track in the counterclockwise direction (black arrow) for food reward given at the two adjacent corners indicated by the white squares. $\boldsymbol{B}$, T-shaped track with moveable barrier. Rats traversed this track for food reward given with differing probabilities at the arm ends. The white squares indicate reward locations, and the black arrows show the rat's trajectory. C, Circular shuttle track. Rats ran back and forth between the start box (black square) and the barrier (black vertical line) for medial forebrain bundle stimulation given at the hidden goal zone on a circular track. Between trials, the location of the start box was rotated (indicated by the black arrow) to vary its distance from the goal zone. The barrier and the goal zone did not move.

\section{Data analysis}

CA1 principal cell isolation. Multidimensional "cluster-cutting" techniques were used to isolate single units on each tetrode [XClust by M. Wilson, MClust by A. D. Redish, and BubbleClust by P. Lipa (Arizona Research Laboratories Division of Neural Systems, Memory and Aging)] based on previously described algorithms (McNaughton et al., 1983a; Wilson and McNaughton, 1993). This method uses the different waveform parameters on the four separate channels of the tetrode probe to discriminate among multiple individual units recorded from a given tetrode. Units were classified as pyramidal cells or interneurons. To be classified as a pyramidal cell, an isolated neuron had to meet the following criteria: (1) it had to fire at least a small number of complex spike bursts during the REST periods, (2) it was recorded on the same tetrode as other complex spike cells in CA1, (3) it had a spike width (peak to trough) of at least $300 \mu \mathrm{s}$, and (4) it had a mean firing rate $<5 \mathrm{~Hz}$ during track running. A pyramidal cell was considered location specific if it had significant $(p<0.01)$ spatial specificity (a place field) during track running. The spatial selectivity was quantified as the amount of information a single spike provides with respect to the rat's location (i.e., information content) (Skaggs et al., 1993). Pyramidal cells that fired only few or no spikes during track running were classified as having no place field in that particular environment. Only the pyramidal cells that were stable across the entire recording session (REST1, RUN, and REST2) were included in the analysis.

Temporal bias analysis. Several methods for measuring the reactivation of hippocampal pyramidal cell activity sequences have been reported previously (Skaggs and McNaughton, 1996; Nadasdy et al., 1999; Lee and Wilson, 2004; Tatsuno et al., 2006). One method for quantifying the reactivation of short temporal sequences (i.e., segments of events replayed in the sample temporal order) uses the cross-correlograms of pyramidal cell pairs that have overlapping place fields to measure the "temporal bias" of cell firing sequences during behavior and sleep (Skaggs and McNaughton, 1996). If two neurons (A and B) in a widely interconnected network have overlapping place fields such that neuron $\mathrm{A}$ fires immediately before neuron $\mathrm{B}$, and this temporal sequence is repeated during track running, then synaptic plasticity mechanisms would be expected to strengthen the theoretical connection from cell A to cell B. Therefore, as one cell repeatedly fires just before the next, this repeated sequence forms a temporal bias in network activity and this temporal asymmetry for neuron A to fire just before neuron B between those two spike trains ( $i$ and $j$ ) is preserved during the postbehavior rest period, and is reflected as an asymmetry in the cross-correlogram of those neurons.

The temporal bias was calculated for each pair of cells active during the RUN epoch by time shifting each spike train relative to the other by $10 \mathrm{~ms}$ intervals from +500 to $-500 \mathrm{~ms}$. The cross-correlation between the two spike trains (neurons) was then calculated and recorded at each interval, producing a cross-correlation histogram from each cell pair recorded from different tetrodes (Skaggs and McNaughton, 1996). The temporal bias or asymmetry was measured as the number of events in which a spike from cell $j$ was followed within $200 \mathrm{~ms}$ by a spike from cell $i$, using the following calculation:

$$
B_{i j}=\int_{j}^{200} x_{i j}(t) d t-\int_{-200}^{0} x_{i j}(t) d t,
$$

where $x_{i j}(t)$ is the cross-correlation value between the cell pair $i$ and $j$ and $\Delta t$ is the time shift $(10 \mathrm{~ms})$. One potential difficulty with the above calculation from Skaggs and McNaughton (1996) is the result can be any real number. Therefore, this value was normalized to produce a result between -1 and 1 , with -1 representing a complete anticorrelation and 1 representing a perfect positive correlation. The final temporal bias was computed as the normalized difference between the $200 \mathrm{~ms}$ window preceding time 0 (PRE) (see Fig. 2, shaded black) and the $200 \mathrm{~ms}$ window following time 0 (POST) (see Fig. 2, shaded gray) using the following equation: (PRE - POST/PRE + POST), where PRE and POST are the result of integrating the cross-correlation histogram over their respective $200 \mathrm{~ms}$ time windows.

Temporal bias: center of mass analysis. In addition to the above normalization of the calculation from Skaggs and McNaughton (1996), a second method for computing temporal bias was conducted by calculating the "center of mass" or first moment within each cell pair cross-correlation histogram. The center of mass for each cross-correlation histogram of the cell pair $i$ and $j$ was calculated within the $\pm 200 \mathrm{~ms}$ time window for each cell pair as follows:

$$
\operatorname{COM}_{i j}=\frac{\sum_{1}^{N} X_{i j}(t) \times t}{\sum_{1}^{N} X_{i j}(t)},
$$

where $X_{i j}(t)$ is the cross-correlation of the cell pair $i j$ at the time bin $t$ and $N$ is the number of time bins within the cross-correlogram. Thus, if cell $i$ has a strong tendency to fire just before cell $j$, then the resulting crosscorrelogram will have a large peak in the region between the -200 to $0 \mathrm{~ms}$ time shift, moving the CENTER OF MASS into this region of the time scale. The center of mass for each cell pair that was active during the RUN epoch was computed for each of the three epochs (REST1, RUN, REST2).

Sequence reactivation: preservation of temporal bias. The temporal bias of each cell pair active during track running (RUN) was calculated. The preservation of the RUN temporal bias (temporal order) during the subsequent off-line period (RUN2) was taken to represent the preservation 
of sequential pyramidal cell activity formed during track running. This sequence reactivation was measured as the correlation of the temporal bias patterns from REST and RUN epochs. The correlation coefficient was computed between the temporal bias patterns of RUN and those of REST1 and REST2. In addition, the partial correlation between the temporal bias pattern in REST2 and RUN, accounting for any preexisting temporal bias pattern in REST1, was also computed.

Inclusion criteria and statistical comparisons in aging data. To compute an appropriate cross-correlation histogram in the temporal bias calculation, the pyramidal cells used for this analysis had to fire an adequate number of spikes during the track-running session (RUN) and during both rest epochs. This criterion eliminates the majority of pyramidal cells recorded during the experiment. First, only $\sim 30-40 \%$ of hippocampal pyramidal cells will fire during any given track-running experience in either age group (Wilson and McNaughton, 1993; Shen et al., 1997). This means $60-70 \%$ of the pyramidal cells recorded were eliminated from the analysis for lack of activity during the RUN epoch. In addition, for each pairwise computation of temporal bias, both neurons must produce enough spikes during the $\pm 200 \mathrm{~ms}$ time window to compute a reliable cross-correlation value. This typically requires that the neurons have overlapping place fields, and therefore cell pairs that did show overlap in the spatial extent of the their firing were not included in this analysis. Furthermore, it has been previously noted that datasets with $<50$ cell pair comparisons were potentially sensitive to strong outliers. Therefore, at least 50 cell pair comparisons were required for each animal in order for that animal to be included in the analysis. Finally, if one animal in a young/old pairing failed to meet inclusion criteria, then its paired animal was also discarded from the analysis. In 2 of the 11 young/old pairings, one or both animals did not meet the above inclusion criteria and were removed from the analysis. In the final analysis, the data from 18 animals, 9 young and 9 old, were included. The average number of cell pairs among the animals remaining in the analysis of temporal bias preservation was $>300$. It is commonly accepted that statistical comparisons between age groups are done using the number of subjects as the $N$ in each group (Barnes, 1994) rather than the number of observations. The age group comparisons in the present study were done on a per-animal basis because a per-cell or per-dataset basis can artificially inflate the degrees of freedom. For example, each neuron recorded during these experiments was active in the structure of a network of neurons within each animal and was not necessarily an independent unit. Thus, multiple measurements from each single animal were averaged to accurately reflect each subject's contribution to the old or young group. Therefore, in the final statistical analysis each animal contributed one number to the statistical results and comparisons. Although $>5000$ cell pairs were analyzed, $N=9$ in the statistical analysis because there were nine animals in each age group.

\section{Results}

\section{Morris swim task}

Repeated-measures ANOVAs were performed on the data from the spatial and visual discrimination trials on the swim task. In the spatial version of the swim task, both age groups showed an improvement over trials $\left(F_{(1,23)}=4.47 ; p<0.001\right)$; however, the old rats spent more time searching for the platform than did the young animals $(F=17.719 ; p<0.01)$ (see Fig. $4 A)$. In the visual discrimination version of the swim task (platform visible or marked), both the young and old rats improved over trials $\left(F_{(1,11)}\right.$ $=2.056 ; p<0.05)$, and there was no effect of age on performance as measured by the latency to the platform $(p>0.10$, ANOVA). Finally, there was no trial by age interaction $\left(F_{(1,11)}=0.222 ; p>\right.$ 0.10 ) in the cued version of the swim task (see Fig. $4 A$ ). These results are consistent with many previous reports of spatial memory deficits in aged rats on the Morris swim task (Gage et al., 1984; Gallagher et al., 1993; Barnes et al., 1997).
A Cell 1

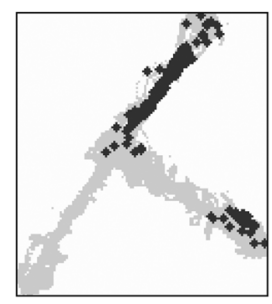

Cell 2

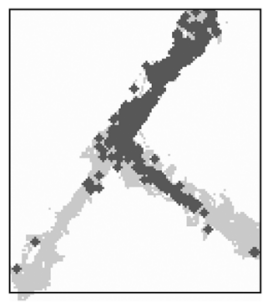

B
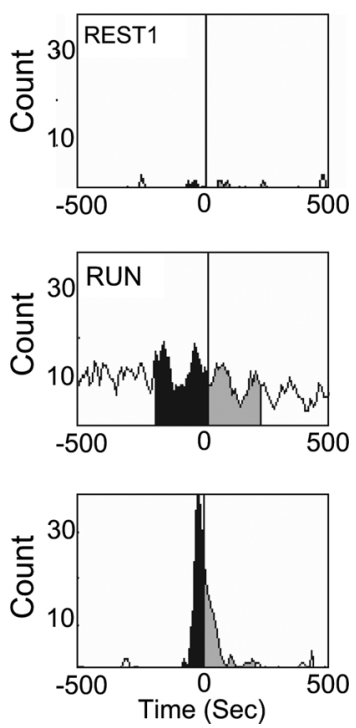

Figure 2. An example of the temporal bias analysis for a single pair of CA1 pyramidal cells with overlapping place fields. $A$, Each spike during track running on the T-shaped track is plotted for the two neurons, cell 1 in black and cell 2 in gray. $\boldsymbol{B}$, The cross-correlogram of this cell pair for REST1 shows little correlated firing within the $1 \mathrm{~s}$ window (top panel). The $y$-axis shows the count for each 10 ms bin shown on $x$-axis. For RUN, strong theta frequency $(\sim 7-8 \mathrm{~Hz})$ modulation is observed in the cross-correlogram, and in this cell pair, cell 2 tends to fire just before cell 1 (middle panel). This tendency is maintained during REST2 as the peak of the crosscorrelogram occurs before zero (bottom panel). The difference in the area under the curve between -200 to $0 \mathrm{~ms}$ (black) and 0 to $200 \mathrm{~ms}$ (gray) for each cross-correlogram is calculated to obtain the temporal bias measure.

\section{Sequence reactivation: temporal bias method}

An example of how the temporal bias of cell pair activity is measured is shown in Figure 2 (for more details, see Materials and Methods). Neither the young animals $(r=0.003 \pm 0.026)$ nor the aged animals $(r=0.048 \pm 0.023)$ showed a significant correlation between the temporal bias patterns in REST1 and the temporal activity patterns during the behavioral epoch $(p>0.20$, one sample $t$ test; for both comparisons) (Fig. 2). The young animals, however, did show a temporal bias correlation between the behavior and REST2 epochs that was significantly greater than zero ( $p<0.01$, one-sample $t$ test) and significantly greater than the REST1-behavior correlation $\left({ }^{\star} p<0.05\right.$, paired $t$ test) (Fig. 3A). This result is consistent with the temporal bias measures obtained by Skaggs and McNaughton (1996) in that the patterns of pyramidal cell firing during the subsequent off-line period (REST2) reflected sequences of cell firing during behavior (RUN). In contrast, the aged animals failed to show significant preservation of the RUN temporal bias during REST2, because the RUN-REST2 temporal bias correlation was not different from zero ( $p>0.10$, one sample $t$ test). Additionally, the RUNREST2 temporal bias correlation was not significantly different from the temporal bias correlation of REST1-RUN in the old animals ( $p>0.20$, paired $t$ test). Finally, there was a significant difference between the age groups in temporal bias (RUN-REST2 correlation, ${ }^{*} p<0.05$, paired $t$ test) (Fig. $3 A$ ), indicating that there is an age-related deficit in sequence reactivation.

\section{Sequence reactivation: center of mass analysis}

The center of mass measure of sequence reactivation (see Materials and Methods) was also used to provide convergent evidence that aged rats may be impaired at reactivating sequences of activ- 

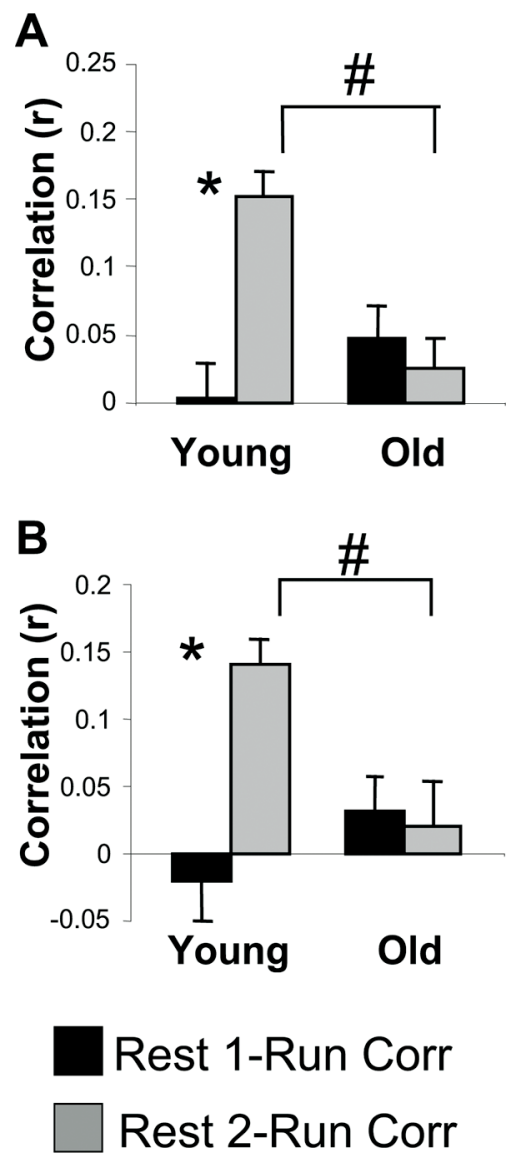

Figure 3. The neuronal firing sequence pattern that is observed during track running is preserved during the subsequent rest period in young animals, but not in old animals. $A$, The mean correlation of the temporal bias measure between REST1 and RUN (black), and REST2 and RUN (gray) in young and old rats. There was no significant correlation of RUN with REST1 in young or in old rats. In young rats only, the REST2-RUN temporal bias correlation was significantly larger than the REST1-RUN correlation ( ${ }^{*} p<0.05$, paired $t$ test). Additionally, there was an effect of age on the REST2-RUN temporal bias correlation, with young rats showing a significantly larger correlation ( ${ }^{\#} p<0.05$, paired $t$ test). $\boldsymbol{B}$, The mean correlation between REST1 and RUN (black) and REST2 and RUN (gray) of the "center of mass" values of the crosscorrelograms. The young group exhibited a significantly higher correlation of the center of mass values between REST2-RUN compared with REST1-RUN ( ${ }^{*} p<0.05$, paired $t$ test). The old rats did not show a significant correlation of center of mass values between REST1-RUN or REST2RUN and had a significantly smaller correlation of REST2-RUN compared with the young animals ( ${ }^{\#} p<0.05$, paired $t$ test). Thus, both analytic methods support the conclusion that the olds rats have defective sequence replay. Error bars indicate SEM.

ity patterns compared with young animals. As in the previous analysis, the center of mass measure indicated that neither age group exhibited a significant relationship between the temporal bias measure in REST1 with those in the track-running period (RUN) (Fig. 2B). That is, the REST1-RUN temporal bias correlations were not significantly different from zero in either age group ( $p>0.20$, one-sample $t$ test). In the young group, the RUN-REST2 correlation in the center of mass distribution was significantly different from zero $(p<0.05$, one-sample $t$ test) and was significantly higher than for the REST1-RUN correlation $\left({ }^{*} p<0.05\right.$, paired $t$ test) (Fig. $\left.3 B\right)$. As before, the mean RUN-REST2 correlation in the aged group was not significantly different from zero ( $p>0.20$, one-sample $t$ test) or from the REST1-RUN temporal bias correlation ( $p>0.20$, paired $t$ test). Finally, the mean RUN-REST2 correlation from the young group was significantly greater than that of the aged group $\left({ }^{\#} p<0.05\right.$, paired $t$ test) (Fig. 3B). Thus, a significant effect of age was found in sequence reactivation using two different methods.

Although the overall mean for the old group did not indicate significant sequence reactivation during REST2, it was noted during the analysis that the deficit was not uniform across all the aged animals. This raises the possibility that an individual animal's behavior may be related to the accurate reactivation of temporal activity patterns. This was tested as reported below.

\section{Sequence reactivation versus Morris swim task performance} The possibility that the preservation of temporal neuronal activity during off-line periods may correlate with spatial memory in rats was investigated by comparing the sequence reactivation results from each animal with the average latency (six trials) from the final day of the spatial trials in the Morris swim task. The trials from the fourth day of this spatial task typically reflect the asymptotic memory for the platform location in young rats (Barnes et al., 1997).

In both age groups, there were obvious trends in the data for a correlation between spatial memory scores and sequence reactivation. Because it is difficult to establish significant neurobehavioral relationships with a small number of animals, this did not reach significance in either group (young, $p=0.11, r^{2}=0.32$; old, $p=0.20, r^{2}=0.18$ ). Therefore, data from both age groups were combined into a single analysis by converting the RUNREST2 temporal bias correlation and the Morris swim task latency into $z$ scores within each age group separately. Thus, the data within each age group were normalized by the distributions of each separate age group. This eliminates the possibility of detecting an artificial relationship attributable to the differences in the mean of these data for the two age groups (Baxter and Gallagher, 1996). A regression was then computed between the two sets of $z$ score data (Fig. $4 \mathrm{~B}$ ). A significant relationship was found between the temporal bias preservation and spatial memory performance $\left(p<0.05 ; r^{2}=0.285\right)$ (Fig. $\left.4 B\right)$. When the same comparison was done with the center of mass calculation, the results were similar. Again, there was a trend in both age groups separately and a significant relationship between sequence reactivation and performance in the spatial version of the Morris swim task $\left(p<0.01 ; r^{2}=0.388\right)$ in the normalized and combined data. These data support the idea that successful sequence reactivation may be related to the accuracy of spatial memory.

\section{Discussion}

The most important findings to result from this experiment are the observations that old rats do not show reliable preservation of the temporal order of activity patterns from previous behavioral experience during subsequent off-line periods of rest and that, regardless of age, animals that show the strongest temporal bias preservation also have the best spatial memory.

It may, at first, seem paradoxical that aged animals show preserved activity pattern reactivation but are impaired at reactivating the appropriate temporal order of these activity sequences. A potential explanation for this dissociation is that simple pattern reactivation depends only on cell pair correlations that are independent of temporal order. Any scrambling of the set of population vectors would have no effect on the magnitude of simple correlations. In contrast, for the sequence reactivation computation, the order of reactivated patterns is critical. In this view, simple activity pattern reactivation would not require the forms of associative plasticity that are impaired in aged animals. In fact, Shen and McNaughton (1996) developed a model indicating that once a synaptic distribution (or map) for several environments is 

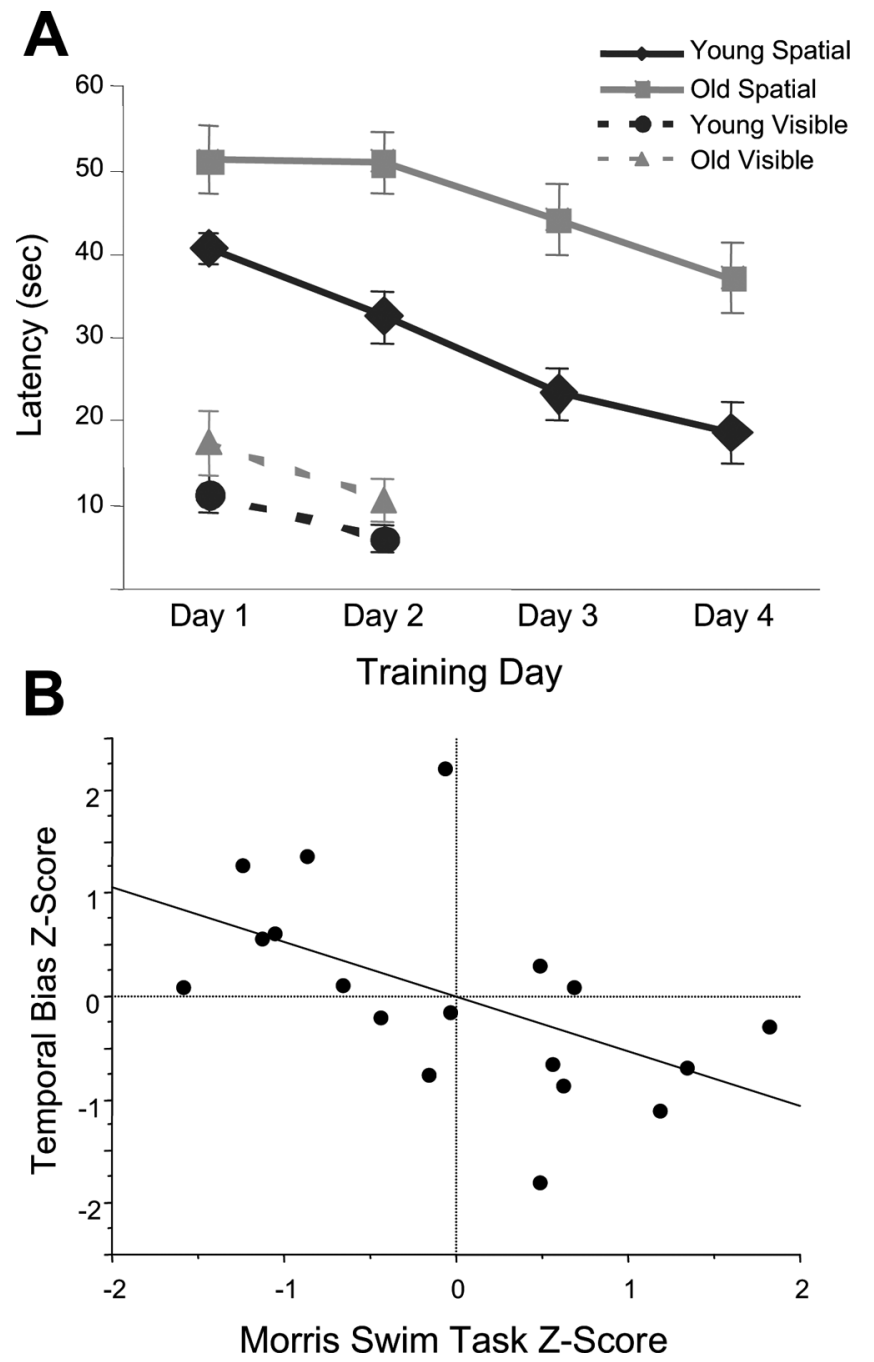

Figure 4. Age comparison of the Morris swim task and correlation of temporal bias scores with spatial behavior. $\boldsymbol{A}$, In the spatial version of the swim task, both age groups showed an improvement over trials (ANOVA; $F_{(1,23)}=4.467 ; p<0.001$ ); however, the old animals spent more time searching for the platform (ANOVA; $F_{(1,23)}=17.719 ; p<0.01$ ). In the visible platform version of the task, no age differences were noted, suggesting comparable motor and visual capacities between groups. Error bars indicate SEM. $\boldsymbol{B}$, The temporal bias scores from each rat were compared with the animal's performance in the spatial version of the Morris swim task. In this plot, the data were normalized to remove age as a factor by computing the $Z$ score for both the temporal bias data and the average latency on the spatial trials of the Morris swim task within each age group separately. There is a significant correlation between preserved temporal bias and spatial memory performance $\left(r^{2}=0.285 ; p<0.05\right)$. These data are consistent with the hypothesis that sequence reactivation is related to memory consolidation processes.

established, the continued increase of pairwise correlations during postbehavior rest (REST2) can be accounted for equally by either associative synaptic modification, or by a bias in the excitability of recently active cells in a network using sparse encoding. Thus, hippocampal activity patterns may reflect the existence of so-called "continuous attractor" states in the network (Skaggs et al., 1995; McNaughton et al., 1996; Zhang, 1996; Samsonovich and McNaughton, 1997). In theory, once a given map is established by external input, the dynamics of the attractor network could allow the network to remain on a given map even after the removal of the input. The pattern reactivation observed in the hippocampus of aged (and perhaps young) animals may reflect the tendency of the network to remain in, or return to one or more dynamically stable regions caused by a nonassociational excitability bias within an existing attractor. In contrast, sequence reactivation may rely on associative changes within the synaptic network that occur during behavioral activity. Specifically, when a young rat repeatedly traverses the same path, there is an asymmetric expansion in the size of the place field and a shift in its center of mass (Mehta et al., 1997, 2000; Shen et al., 1997; Ekstrom et al., 2001; Lee et al., 2004; Burke et al., 2008). In most cases, the shift is opposite to the direction of the rat's trajectory, although under some circumstances the place field can also shift forward (Lee et al., 2006; Lee and Knierim, 2007). This behaviorally induced center of mass shift in the location of place cell firing supports the idea that the occurrence of asymmetric synaptic changes can result from behavioral activity. Importantly, aged rats show impaired experience-dependent place field expansion plasticity (Shen et al., 1997; Burke et al., 2008). This, in fact, is one possible explanation that might be offered to account for the observed impairment in sequence reactivation. An alternative explanation is that neither pattern nor sequence reactivation requires associative plasticity, but rather the excitability bias established during behavior is sufficient to induce these patterned states. In this view, sequence reactivation would occur via the inherent asymmetry in the hippocampal network that also supports theta phase precession (Jensen and Lisman, 1996; Tsodyks et al., 1996). Because this proposed asymmetry can be established by the CA3 to CA1 Schaffer collateral synapse, it is possible that, with aging, functional changes that occur at this synapse (Landfield et al., 1978; Barnes et al., 1992, 2000; Geinisman et al., 2004; Nicholson et al., 2004) (for review, see Burke and Barnes, 2006) could result in the observed altered network properties.

One issue raised by the temporal bias results in this study involves that of spike sorting and temporal sequence calculation. Inaccurate spike sorting techniques could potentially lead to artificial cell pair correlations, altering true temporal relationships (Quirk and Wilson, 1999). During quiet rest and sleep, hippocampal pyramidal cells typically fire in complex spike bursts (Fox and Ranck, 1975; McNaughton et al., 1983b), which are commonly associated with sharp wave/ripple events within the hippocampus. During these complex spike bursts, the last spikes in the burst tend to be of lower amplitude than the earlier spikes. Quirk and Wilson (1999) suggested that systematic changes in spike amplitude during burst firing activity make it possible for the low-amplitude spikes from one neuron to blend with highamplitude spikes from another neuron. The possibility of minor spike misclassifications as the explanation for the aged differences in this study seems unlikely. Given that the membrane potential and action potential amplitudes are similar in young and old rats (Barnes, 1994; Shen and Barnes, 1996), it is improbable that there was a systematic and recurrent spike classification error within one age group, but not the other.

An additional issue raised by the current finding that advanced age results in an impairment in sequence reactivation is whether these results could be accounted for by age-related changes in sleep architecture (Stone and Gold, 1988; Mendelson and Bergman 1999). Specifically, in some old rats there is a reduction in the time spent in slow-wave sleep and rapid eye movement (i.e., paradoxical sleep) with advanced age when the EEG is recorded during the light phase of the rat's light/dark cycle. We believe that this is not a confound in the present study, however, for several reasons. Among these is the well documented observation that activity pattern reactivation (Kudrimoti et la., 1999; Gerrard et al., 2001) and sequence reactivation (Nadasdy et al., 1999; Lee and Wilson, 2002) in the hippocampus are concentrated during sharp wave/ripple events. Sharp wave/ripples occur 
both during slow-wave sleep and during quiet wakefulness (Buzsáki et al., 1983). The majority of data that suggest agerelated sleep alterations have been obtained during the light portion of the rat's cycle when the animal is the least active. In the current study, all recording occurred during the dark cycle (i.e., when the lights were out in the animal's colony room and the animal shows more active behavior). Critically, under these conditions, it has been carefully documented that there is no agerelated difference in the occurrence, frequency, or amplitude characteristics of sharp wave/ripple events (Gerrard et al., 2001). Thus, under the recording conditions of these experiments, the process in large-amplitude irregular activity or slow-wave sleep that carries the reactivated signals is not different between age groups, and thus is not likely to be responsible for the observed age-related deficit in sequence reactivation. Furthermore, if agerelated changes in sleep dynamics were responsible for the decline in sequence reactivation, then presumably it would also disrupt simple pattern reactivation. Because activity pattern reactivation does not change during advanced age (Gerrard et al., 2001), it is unlikely that the global differences in the dynamic processes involved in the two rest epochs can fully account for the age difference in the temporal order of these patterns.

In theories of memory consolidation, the hippocampus has been hypothesized to play a critical role in the off-line processing of newly formed memories (Marr, 1971; Squire, 1992; Alvarez et al., 1995; McClelland et al., 1995; Nadel and Moscovitch, 1997, 1998; Nadel and Land, 2000). More specifically, the reactivation or persistence of hippocampal "maps" has been proposed to orchestrate the coherent retrieval of relevant neocortical activity patterns (Treves and Rolls, 1994; McClelland et al., 1995; Buzsáki, 1998; Hasselmo and McClelland, 1999) during periods in which the hippocampus is off-line. The observations that aged rats store sequentially ordered neural activity less reliably within the hippocampal map, and show defective place field expansion (Shen et al., 1997), are both consistent with the temporal bias defects found in this study. Additional support for the idea that these changes could contribute to aged-related learning and memory impairments comes from the significant correlation between sequence reactivation and spatial memory scores. These data are the first to indicate that there may be behavioral consequences of weakened replay of temporal sequences, and are consistent with the hypothesis that the reactivation process contributes to a mechanism for memory consolidation.

\section{References}

Alvarez P, Zola-Morgan S, Squire LR (1995) Damage limited to the hippocampal region produces long-lasting memory impairment in monkeys. J Neurosci 15:3796-3807.

Barnes CA (1979) Memory deficits associated with senescence: a neurophysiological and behavioral study in the rat. J Comp Physiol Psychol 93:74-104.

Barnes CA (1994) Normal aging: regionally specific changes in hippocampal synaptic transmission. Trends Neurosci 17:13-18.

Barnes CA, Rao G, Foster TC, McNaughton BL (1992) Region-specific age effects on AMPA sensitivity: electrophysiological evidence for loss of synaptic contacts in hippocampal field CA1. Hippocampus 2:457-468.

Barnes CA, Suster MS, Shen J, McNaughton BL (1997) Multistability of cognitive maps in the hippocampus of old rats. Nature 388:272-275.

Barnes CA, Rao G, Orr G (2000) Age-related decrease in the Schaffer collateral-evoked EPSP in awake, freely behaving rats. Neural Plast 7:167-178.

Baxter MG, Gallagher M (1996) Neurobiological substrates of behavioral decline: models and data analytic strategies for individual differences in aging. Neurobiol Aging 17:491-495.

Bolhuis JJ, Stewart CA, Forrest EM (1994) Retrograde amnesia and memory reactivation in rats with ibotenate lesions to the hippocampus or subiculum. Q J Exp Psychol B 47:129-150.

Burke SN, Barnes CA (2006) Neural plasticity in the ageing brain. Nat Rev Neurosci 7:30-40.

Burke SN, Maurer AP, Yang Z, Navratilova Z, Barnes CA (2008) Glutamate receptor-mediated restoration of experience-dependent place field expansion plasticity in aged rats. Behav Neurosci 122:535-548.

Buzsáki G (1986) Hippocampal sharp waves: their origin and significance. Brain Res 398:242-252.

Buzsáki G (1998) Memory consolidation during sleep: a neurophysiological perspective. J Sleep Res 7 [Suppl 1]:17-23.

Buzsáki G, Leung LW, Vanderwolf CH (1983) Cellular bases of hippocampal EEG in the behaving rat. Brain Res 287:139-171.

Ekstrom AD, Meltzer J, McNaughton BL, Barnes CA (2001) NMDA receptor antagonism blocks experience-dependent expansion of hippocampal "place fields." Neuron 31:631-638.

Euston DR, Tatsuno M, McNaughton BL (2007) Fast-forward playback of recent memory sequences in prefrontal cortex during sleep. Science 318:1147-1150.

Fox SE, Ranck JB Jr (1975) Localization and anatomical identification of theta and complex spike cells in dorsal hippocampal formation of rats. Exp Neurol 49:299-313.

Gage FH, Dunnett SB, Björklund A (1984) Spatial learning and motor deficits in aged rats. Neurobiol Aging 5:43-48.

Gallagher M, Rapp PR (1997) The use of animal models to study the effects of aging on cognition. Annu Rev Psychol 48:339-370.

Gallagher M, Burwell R, Burchinal M (1993) Severity of spatial learning impairment in aging: development of a learning index for performance in the Morris water maze. Behav Neurosci 107:618-626.

Geinisman Y, Ganeshina O, Yoshida R, Berry RW, Disterhoft JF, Gallagher M (2004) Aging, spatial learning, and total synapse number in the rat CA1 stratum radiatum. Neurobiol Aging 25:407-416.

Gerrard JL, Kudrimoti H, McNaughton BL, Barnes CA (2001) Reactivation of hippocampal ensemble activity patterns in the aging rat. Behav Neurosci 115:1180-1192.

Gothard KM, Skaggs WE, Moore KM, McNaughton BL (1996) Binding of hippocampal CA1 neural activity to multiple reference frames in a landmark-based navigation task. J Neurosci 16:823-835.

Hasselmo ME, McClelland JL (1999) Neural models of memory. Curr Opin Neurobiol 9:184-188.

Hoffman KL, McNaughton BL (2002) Coordinated reactivation of distributed memory traces in primate neocortex. Science 297:2070-2073.

Jensen O, Lisman JE (1996) Hippocampal CA3 region predicts memory sequences: accounting for the phase precession of place cells. Learn Mem 3:279-287.

Ji D, Wilson MA (2007) Coordinated memory replay in the visual cortex and hippocampus during sleep. Nat Neurosci 10:100-107.

Kudrimoti HS, Barnes CA, McNaughton BL (1999) Reactivation of hippocampal cell assemblies: effects of behavioral state, experience, and EEG dynamics. J Neurosci 19:4090-4101.

Landfield PW, McGaugh JL, Lynch G (1978) Impaired synaptic potentiation processes in the hippocampus of aged, memory-deficient rats. Brain Res 150:85-101.

Lee AK, Wilson MA (2002) Memory of sequential experience in the hippocampus during slow wave sleep. Neuron 36:1183-1194.

Lee AK, Wilson MA (2004) A combinatorial method for analyzing sequential firing patterns involving an arbitrary number of neurons based on relative time order. J Neurophysiol 92:2555-2573.

Lee I, Knierim JJ (2007) The relationship between the field-shifting phenomenon and representational coherence of place cells in CA1 and CA3 in a cue-altered environment. Learn Mem 14:807-815.

Lee I, Rao G, Knierim JJ (2004) A double dissociation between hippocampal subfields: differential time course of CA3 and CA1 place cells for processing changed environments. Neuron 42:803-815.

Lee I, Griffin AL, Zilli EA, Eichenbaum H, Hasselmo ME (2006) Gradual translocation of spatial correlates of neuronal firing in the hippocampus toward prospective reward locations. Neuron 51:639-650.

Markowska AL, Stone WS, Ingram DK, Reynolds J, Gold PE, Conti LH, Pontecorvo MJ, Wenk GL, Olton DS (1989) Individual differences in aging: behavioral and neurobiological correlates. Neurobiol Aging 10:31-43. 
Marr D (1971) Simple memory: a theory for archicortex. Philos Trans R Soc Lond B Biol Sci 262:23-81.

McClelland JL, McNaughton BL, O'Reilly RC (1995) Why there are complementary learning systems in the hippocampus and neocortex: insights from the successes and failures of connectionist models of learning and memory. Psychol Rev 102:419-457.

McNaughton BL, O'Keefe J, Barnes CA (1983a) The stereotrode: a new technique for simultaneous isolation of several single units in the central nervous system from multiple unit records. J Neurosci Methods 8:391-397.

McNaughton BL, Barnes CA, O'Keefe J (1983b) The contributions of position, direction, and velocity to single unit activity in the hippocampus of freely-moving rats. Exp Brain Res 52:41-49.

McNaughton BL, Barnes CA, Gerrard JL, Gothard K, Jung MW, Knierim JJ, Kudrimoti H, Qin Y, Skaggs WE, Suster M, Weaver KL (1996) Deciphering the hippocampal polyglot: the hippocampus as a path integration system. J Exp Biol 199:173-185.

McNaughton BL, Barnes CA, Battaglia FP, Bower MR, Cowen SL, Ekstrom AD, Hoffman KL, Houston FP, Karten Y, Lipa P, Pennartz CM, Sutherland GR (2003) Off-line reprocessing of recent memory and its role in memory consolidation: a progress report. In: Sleep and brain plasticity (Stickgold B, ed), pp 225-246. Oxford: Oxford UP.

Mehta MR, Barnes CA, McNaughton BL (1997) Experience-dependent, asymmetric expansion of hippocampal place fields. Proc Natl Acad Sci U S A 94:8918-8921.

Mehta MR, Quirk MC, Wilson MA (2000) Experience-dependent asymmetric shape of hippocampal receptive fields. Neuron 25:707-715.

Mendelson WB, Bergmann BM (1999) Age-related changes in sleep in the rat. Sleep 22:145-150.

Nádasdy Z, Hirase H, Czurkó A, Csicsvari J, Buzsáki G (1999) Replay and time compression of recurring spike sequences in the hippocampus. J Neurosci 19:9497-9507.

Nadel L, Land C (2000) Memory traces revisited. Nat Rev Neurosci $1: 209-212$.

Nadel L, Moscovitch M (1997) Memory consolidation, retrograde amnesia and the hippocampal complex. Curr Opin Neurobiol 7:217-227.

Nadel L, Moscovitch M (1998) Hippocampal contributions to cortical plasticity. Neuropharmacology 37:431-439.

Nicholson DA, Yoshida R, Berry RW, Gallagher M, Geinisman Y (2004) Reduction in size of perforated postsynaptic densities in hippocampal axospinous synapses and age-related spatial learning impairments. J Neurosci 24:7648-7653.

O’Keefe J, Recce ML (1993) Phase relationship between hippocampal place units and the EEG theta rhythm. Hippocampus 3:317-330.

Olds J, Milner P (1954) Positive reinforcement produced by electrical stimulation of septal area and other regions of rat brain. J Comp Physiol Psychol 47:419-427.

Paxinos G, Watson C (1998) The rat brain in stereotaxic coordinates, Ed 4 San Diego: Academic.

Qin YL, McNaughton BL, Skaggs WE, Barnes CA (1997) Memory reprocessing in corticocortical and hippocampocortical neuronal ensembles. Philos Trans R Soc Lond B Biol Sci 352:1525-1533.

Quirk MC, Wilson MA (1999) Interaction between spike waveform classification and temporal sequence detection. J Neurosci Methods 94:41-52.

Redish AD, Rosenzweig ES, Bohanick JD, McNaughton BL, Barnes CA (2000) Dynamics of hippocampal ensemble activity realignment: time versus space. J Neurosci 20:9298-9309.

Rosenzweig ES, Redish AD, McNaughton BL, Barnes CA (2003) Hippocampal map realignment and spatial learning. Nat Neurosci 6:609-615
Samsonovich A, McNaughton BL (1997) Path integration and cognitive mapping in a continuous attractor neural network model. J Neurosci 17:5900-5920.

Scoville WB, Milner B (1957) Loss of recent memory after bilateral hippocampal lesions. J Neurochem 20:11-21.

Shen B, McNaughton BL (1996) Modeling the spontaneous reactivation of experience-specific hippocampal cell assembles during sleep. Hippocampus 6:685-692.

Shen J, Barnes CA (1996) Age-related decrease in cholinergic synaptic transmission in three hippocampal subfields. Neurobiol Aging 17:439-451.

Shen J, Barnes CA, McNaughton BL, Skaggs WE, Weaver KL (1997) The effect of aging on experience-dependent plasticity of hippocampal place cells. J Neurosci 17:6769-6782.

Shen J, Kudrimoti HS, McNaughton BL, Barnes CA (1998) Reactivation of neuronal ensembles in hippocampal dentate gyrus during sleep after spatial experience. J Sleep Res 7 [Suppl 1]:6-16.

Skaggs WE, McNaughton BL (1996) Replay of neuronal firing sequences in rat hippocampus during sleep following spatial experience. Science 271:1870-1873.

Skaggs WE, McNaughton BL, Gothard KG, Markus EJ (1993) An information theoretic approach to deciphering the hippocampal code. In: Progress in neural information processing systems (Giles CL, ed), pp 1030-1037. San Mateo, CA: Kauffman.

Skaggs WE, Knierim JJ, Kudrimoti HS, McNaughton BL (1995) A model of the neural basis of the rat's sense of direction. In: Advances in neural information processing systems (Leen T, ed), pp 130-180. Cambridge, MA: MIT.

Squire LR (1992) Memory and the hippocampus: a synthesis from findings with rats, monkeys, and humans. Psychol Rev 99:195-231.

Stone WS, Gold PE (1988) Sleep and memory relationships in intact old and amnestic young rats. Neurobiol Aging 9:719-727.

Sutherland GR, McNaughton B (2000) Memory trace reactivation in hippocampal and neocortical neuronal ensembles. Curr Opin Neurobiol 10:180-186.

Takehara K, Kawahara S, Takatsuki K, Kirino Y (2002) Time-limited role of the hippocampus in the memory for trace eyeblink conditioning in mice. Brain Res 951:183-190.

Takehara K, Kawahara S, Kirino Y (2003) Time-dependent reorganization of the brain components underlying memory retention in trace eyeblink conditioning. J Neurosci 23:9897-9905.

Tatsuno M, Lipa P, McNaughton BL (2006) Methodological considerations on the use of template matching to study long-lasting memory trace replay. J Neurosci 26:10727-10742.

Treves A, Rolls ET (1994) Computational analysis of the role of the hippocampus in memory. Hippocampus 4:374-391.

Tsodyks MV, Skaggs WE, Sejnowski TJ, McNaughton BL (1996) Population dynamics and theta rhythm phase precession of hippocampal place cell firing: a spiking neuron model. Hippocampus 6:271-280.

Wilson MA, McNaughton BL (1993) Dynamics of the hippocampal ensemble code for space. Science 261:1055-1058.

Wilson MA, McNaughton BL (1994) Reactivation of hippocampal ensemble memories during sleep. Science 265:676-679.

Winocur G (1990) Anterograde and retrograde amnesia in rats with dorsal hippocampal or dorsomedial thalamic lesions. Behav Brain Res 38:145-154.

Zhang K (1996) Representation of spatial orientation by the intrinsic dynamics of the head-direction cell ensemble: a theory. J Neurosci 16:2112-2126.

Zola-Morgan SM, Squire LR (1990) The primate hippocampal formation: evidence for a time-limited role in memory storage. Science 250:288-290. 